\title{
Stereotactic robot-assisted MRI-guided laser thermal ablation of radiation necrosis in the posterior cranial fossa: technical note
}

\author{
Alvin Y. Chan, BS, ${ }^{2}$ Diem Kieu T. Tran, MD, ${ }^{1}$ Amandip S. Gill, MD, ${ }^{1}$ Frank P. K. Hsu, MD, PhD, ${ }^{1}$ and \\ Sumeet Vadera, MD'
}

${ }^{1}$ Department of Neurological Surgery, University of California, Irvine, California; and 2Department of Neurosurgery, Medical College of Wisconsin, Milwaukee, Wisconsin

\begin{abstract}
Laser interstitial thermal therapy (LITT) is a minimally invasive procedure used to treat a variety of intracranial lesions. Utilization of robotic assistance with stereotactic procedures has gained attention due to potential for advantages over conventional techniques. The authors report the first case in which robot-assisted MRI-guided LITT was used to treat radiation necrosis in the posterior fossa, specifically within the cerebellar peduncle. The use of a stereotactic robot allowed the surgeon to perform LITT using a trajectory that would be extremely difficult with conventional arc-based techniques.

A 60-year-old man presented with facial weakness and brainstem symptoms consistent with radiation necrosis. He had a history of anaplastic astrocytoma that was treated with CyberKnife radiosurgery 1 year prior to presentation, and he did well for 11 months until his symptoms recurred. The location and form of the lesion precluded excision but made the patient a suitable candidate for LITT. The location and configuration of the lesion required a trajectory for LITT that was too low for arc-based stereotactic navigation, and thus the ROSA robot (Medtech) was used. Using preoperative MRI acquisitions, the lesion in the posterior fossa was targeted. Bone fiducials were used to improve accuracy in registration, and the authors obtained an intraoperative CT image that was then fused with the MR image by the ROSA robot. They placed the laser applicator and then ablated the lesion under real-time MR thermometry. There were no complications, and the patient tolerated the procedure well. Postoperative 2-month MRI showed complete resolution of the lesion, and the patient had some improvement in symptoms.
\end{abstract}

http://thejns.org/doi/abs/10.3171/2016.4.FOCUS1622

KEY WORDS infratentorial LITT; laser interstitial thermal therapy; minimally invasive; ROSA; robotic assistance

$\mathrm{L}$ ASER interstitial thermal therapy (LITT) is a stereotactic percutaneous minimally invasive procedure that is popular for treating indicated benign and malignant intracranial lesions. ${ }^{11}$ Although excision is typically first-line therapy for many lesions, ${ }^{17}$ LITT has been improving, allowing for the treatment of a variety of diseases. For example, technological developments allow for the use of MRI guidance that enables surgeons to monitor LITT during the operation (i.e., in real time). ${ }^{11}$ MRI-guided LITT has been used in a number of different procedures, including ablation of epileptogenic lesions, ${ }^{5}$ insular metastatic lesions, ${ }^{6}$ and recurrent glioblastomas, ${ }^{3}$ as well as radiation necrosis. ${ }^{13,14}$ Recent evidence suggests that LITT could treat difficult-to-reach lesions in supratentorial regions, ${ }^{12}$ but LITT treatment for lesions in the posterior fossa has not been as well documented.

Accuracy and precision of the placement of the laser fiber are crucial to the success of any LITT procedure. Robotic assistance has been increasingly used in stereotactic minimally invasive procedures because of its potential for increasing accuracy and precision. ${ }^{10}$ Neurosurgical robots have assisted in a number of cranial procedures, including resection of epileptogenic lesions, ${ }^{5}$ biopsies, ${ }^{8}$ and deep brain stimulation. ${ }^{9}$ The use of robots in intracranial stereotactic procedures is typically accurate and is safe for the patient. ${ }^{2}$ Despite the recent expansion in the use of robotic assistance, it has rarely been described in minimally invasive procedures in the posterior fossa.

Here we present, to our knowledge, the first use of an FDA-approved intracranial robot (ROSA, Medtech) for LITT of a posterior fossa lesion consistent with radiation necrosis. The robot gave us a technical advantage over traditional methods because the tumor required a low entry point (i.e., below standard Leksell head frame placement) to achieve the desired trajectory, which usually prevents the use of a traditional stereotactic arc because the trajectory can be limited to regions above the head frame. Although there have been instances in which thermal ablation 
was used to treat lesions in the posterior fossa, ${ }^{4}$ this is the first known instance in which robotic assistance was used.

\section{Case Report}

History and Presentation

The patient was a 60-year-old man with a history of anaplastic astrocytoma that had been treated using radiosurgery 11 months prior to presentation. During the last month prior to surgery, he developed dysarthria and rightsided cranial nerve V, VI, and VII palsies; MR spectroscopy indicated possible radiation necrosis in the right cerebellar peduncle abutting the pons (Fig. 1A). The patient was put on temozolomide and high doses of Decadron, but he continued to experience symptoms. LITT was discussed as treatment instead of resection because the lesion was determined to be in a difficult location to resect and the potential for increasingly worse symptoms was significant. Additionally, the lesion was amendable to LITT due to its tubular shape.

\section{Treatment}

A preoperative MRI study was uploaded to the ROSA robot, and a safe trajectory between the entry point and target area that avoided major sinuses was planned. The patient underwent general anesthesia and his head was placed in a Leksell frame (Elekta, Inc.) that was fixed to the ROSA robot. The Leksell frame was preferred because it was compatible with the robot (the frame was not used for navigation). The patient was then flipped into a prone position. Skull fiducials were placed around the right posterior fossa in the suboccipital region (Fig. 2) before an intraoperative CT scan was acquired, which was used for robotic navigation and registration. The robot, which consisted of an arm and computer screen, was locked into position once a satisfactory registration was achieved (fewer than $2 \mathrm{~mm}$ in this case).

The entry site was prepared before an incision at the entry point was made (Fig. 1). Because of the location of the laser cannula ablation placement and the thickness of the muscle within this region, an open cut down to the bone had to be performed. With assistance from the robot, a hole was drilled (approximately $1.65 \mathrm{~mm}$ in diameter) through the bone at the entry site, exposing the dura mater. Once the site was coagulated, a needle biopsy of the lesion was performed and the sample was sent for analysis. The pathological diagnosis was radiation necrosis with sparse cells of high-grade glioma.

The Visualase Thermal Therapy System (Medtronic) consists of a 15-W, 980-nm wavelength diode laser, a cooling pump, and a disposable laser applicator set.7.19 A skull bolt was placed and secured into the entry point. A cannula was passed through the bold incision and advanced to the predetermined depth. The laser was then placed within the cannula and secured. The patient was then taken while intubated to the MRI suite for the remainder of the procedure.

After confirmation of the proper laser applicator position, ablation of the lesion commenced. Designated "safety points" within the system would shut off the laser if the protected cerebral structures reached temperatures of

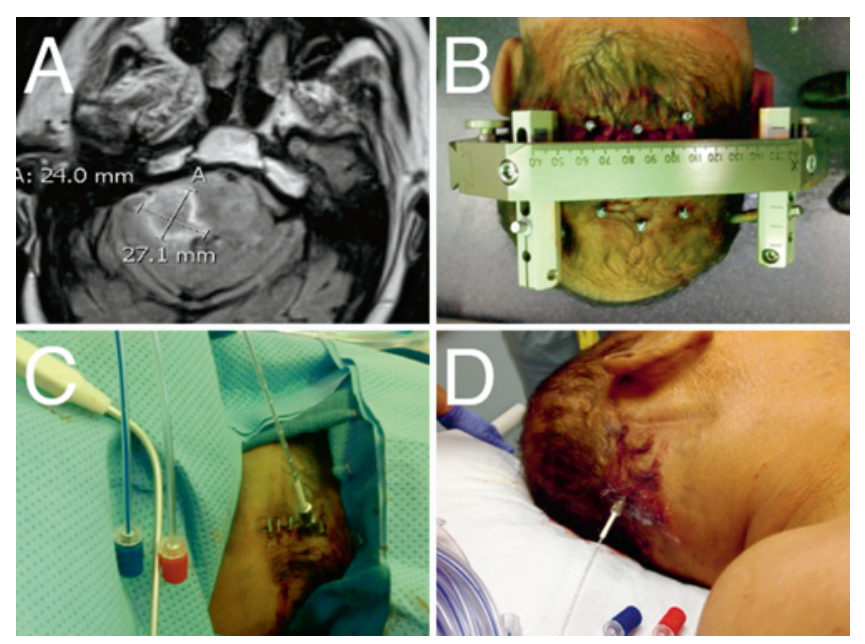

FIG. 1. A: Preoperative axial MR image showing infratentorial lesion. B: The patient in the Leksell head frame with skull fiducials in place. C: Insertion of the laser cannula and bolt intraoperatively. D: The patient being placed within the MRI scanner.

$50^{\circ} \mathrm{C}$, or if an exceedingly high temperature (e.g., $90^{\circ} \mathrm{C}$ ) was reached near the laser applicator or surrounding parenchyma. The laser was pulsed at approximately $9 \mathrm{~W}$ for 60- to 90-second intervals (see Fig. 2). After the lesion was adequately ablated, the laser cannula and bolt were removed and the wound was closed completely.

\section{Postoperative Course}

The patient tolerated the procedure well. There were no immediate complications. He was extubated and taken to the postanesthesia care unit postoperatively in stable condition. He had no adverse events related to the surgery and was discharged the next day without complications. Immediately after surgery, his steroid dosage was increased and then slowly tapered over 2 weeks. Two months postoperatively, imaging showed complete ablation of the lesion (Fig. 3). The patient has had significant improvement in his neurological status, with improved facial nerve function and improved strength in his right arm and leg. His Decadron requirement was decreased, and he stated improvement in headaches. At 4 months, the patient stated that he is still seeing gradual improvements.

\section{Discussion}

This is the first known instance in which robot-assisted LITT was used to ablate radiation necrosis and a highgrade glioma in the posterior cranial fossa. One of the main reasons the robot provided a technical advantage was that the best course of treatment required an unusually low trajectory that fell below the 3D space above the plane of the arc, which made conventional modalities more difficult due to potentially unreliable and inaccurate coordinates. However, by using the ROSA robot, we were able to perform the procedure in an "arc-less" manner and use an optimal trajectory to treat the lesion. While there are other methods that could have been considered (e.g., Brainlab VarioGuide, Clear Point, etc.), this study showed that robotic assistance was possible. 

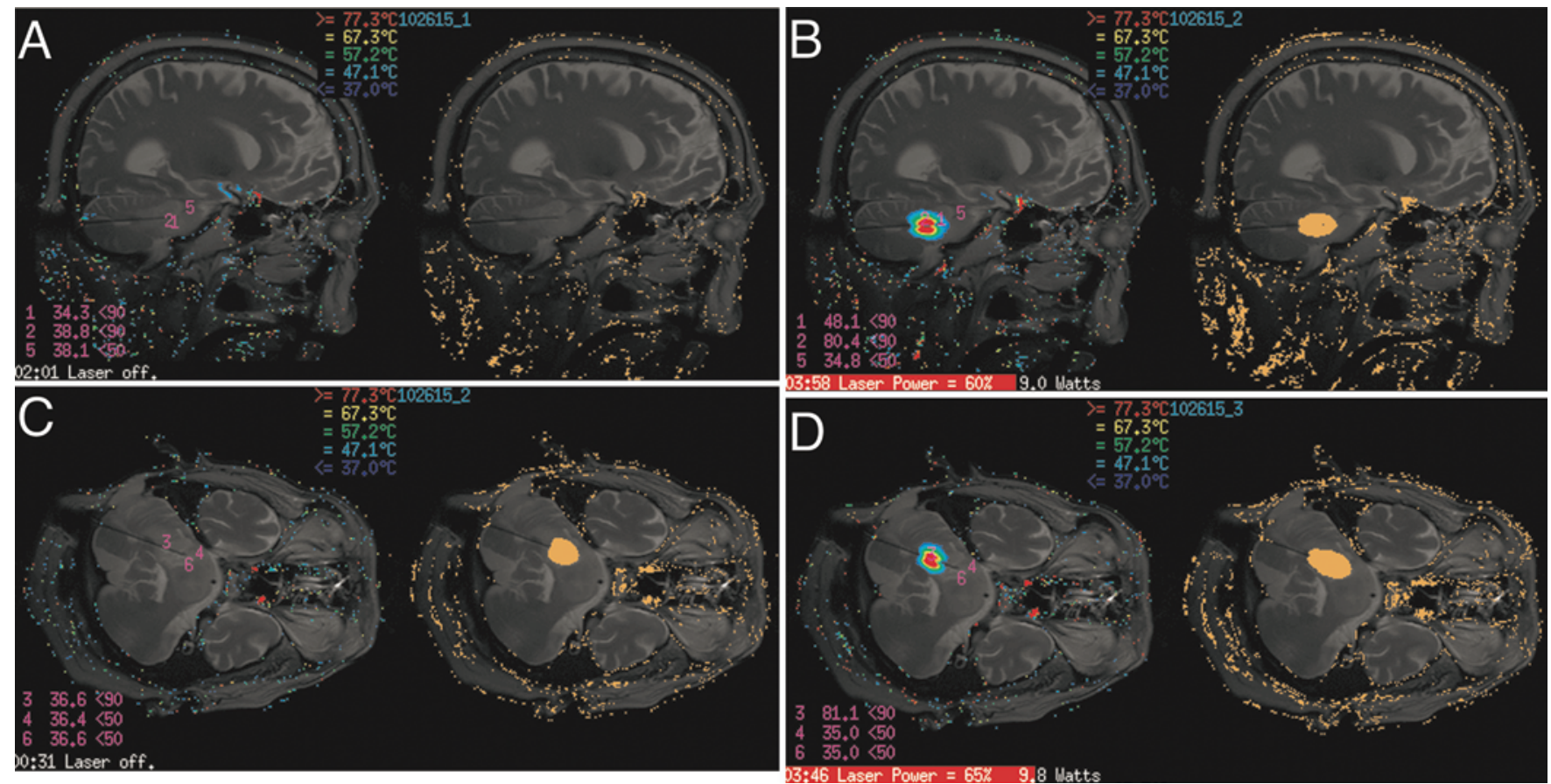

FIG. 2. Real-time tracking of ablation in the posterior fossa. A: Locations $(1,2,5)$ of temperature recording in the sagittal view. B: Temperature readings during ablation in locations $(1,2,5)$ indicating highest temperature at the point of the lesion $\left(80.4^{\circ} \mathrm{C}\right)$. C: Locations $(3,4,6)$ of recording in the axial view. D: Temperature readings during ablation in locations $(3,4,6)$ indicating highest temperature at the point of the lesion $\left(81.1^{\circ} \mathrm{C}\right)$.

Other advantages of using the ROSA robot were its high accuracy, precision, and consistency while also maintaining patient safety. The robot calculated all the trajectories and distances without human intervention, which prevented human or translational errors and likely increased accuracy and thus patient safety. Indeed, our experience at the University of California, Irvine, has been that the robot provides accurate results, which was consistent with the assessment made at several other centers that use robotic stereotactic procedures. ${ }^{5}$ Furthermore, recent evidence has shown that robot assistance in stereotactic procedures has been a safe alternative to conventional techniques. ${ }^{1}$ The ROSA robot allowed for the incorporation of registration and navigation into one system that likely streamlined an efficient procedural workflow. Lastly, one of the major advantages of using the robot was that minor adjustments could be made to the entry point within a 3D plane, unlike conventional arcbased approaches that would have relied on complete readjustment for movements outside of a singular fixed plane. This flexibility in adjustment could reduce some delays that accompany conventional arc-based techniques.

Recent evidence has shown that LITT is a viable option for treating radiation necrosis. ${ }^{13,14}$ Radiation therapy has been an integral part of treating certain cancers like highgrade gliomas, and a risk was the development of radiation necrosis. ${ }^{18}$ Potential therapies include medicine (e.g., high doses of cortical steroids or monoclonal antibodies ${ }^{16}$ ) and resection; for patients who are not strong candidates for surgery and who have medically refractory radiation necrosis, LITT is potentially an alternative. ${ }^{7,13}$ For instance, Torres-Reveron et al. used LITT to successfully treat two cases of cerebellar lesions in which one patient tested positive for tumor recurrence and the other for radiation necrosis. ${ }^{19}$ Our report provides further evidence that LITT is a viable treatment option when more conventional therapies are contraindicated.

Using robotic assistance requires alterations to the conventional workflow. The ROSA robot requires skull fiducials to complete registration, which likely gives it a technical advantage in terms of accuracy and precision over other laser-guided navigational systems that are routinely used in stereotactic procedures. However, this requires additional time and imaging (e.g., CT), which is uploaded to the robot to fuse with the MR image. Finally, the addition of robotic guidance and skull fiducial application increases the length of time for the surgery but also allows for a high level of accuracy, which is required for laser ablations this close to the brainstem and cranial nerves.

\section{Conclusions}

Robotic stereotactic surgery is becoming more popularized in neurosurgery, especially for stereoelectroencephalography. Other navigation/trajectory planning systems such as Brainlab VarioGuide or arc-based targeting strategies are more commonly used by neurosurgeons for stereotactic procedures and the benefits and drawbacks of each system have been well described..$^{15}$ The purpose of this study is to demonstrate a novel means to perform posterior fossa laser ablation of radiation necrosis using robotic stereotactic guidance. We feel that the combination of laser, robot, and posterior fossa radiation necrosis is novel and will likely provide neurosurgeons with an alternative approach to treat this type of pathology. Future 


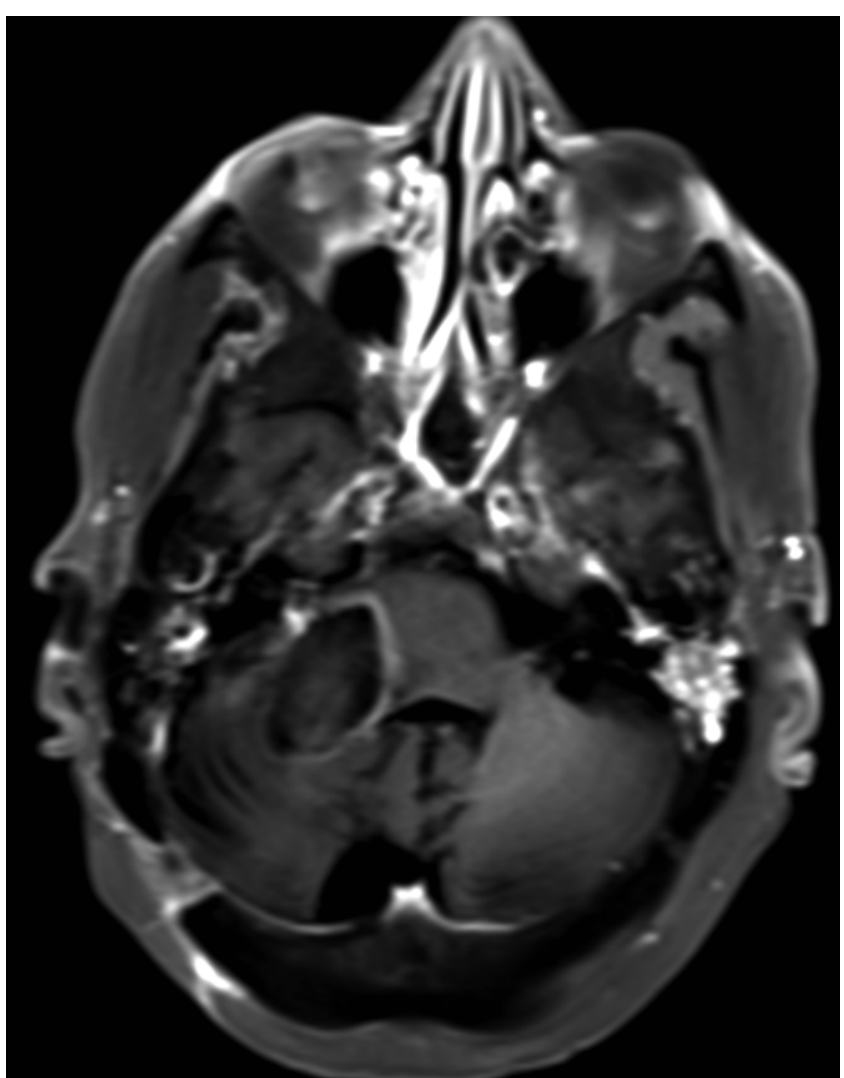

FIG. 3. Axial MR image acquired 2 months postoperatively showing resolution of the enhancing lesion.

studies will be required to assess how robotic treatment options compare with other techniques in terms of accuracy, efficacy, and time required.

\section{References}

1. Bekelis K, Radwan TA, Desai A, Roberts DW: Frameless robotically targeted stereotactic brain biopsy: feasibility, diagnostic yield, and safety. J Neurosurg 116:1002-1006, 2012

2. Cardinale F, Cossu M, Castana L, Casaceli G, Schiariti MP, Miserocchi A, et al: Stereoelectroencephalography: surgical methodology, safety, and stereotactic application accuracy in 500 procedures. Neurosurgery 72:353-366, 2013

3. Carpentier A, Chauvet D, Reina V, Beccaria K, Leclerq D, McNichols RJ, et al: MR-guided laser-induced thermal therapy (LITT) for recurrent glioblastomas. Lasers Surg Med 44:361-368, 2012

4. Eliyas JK, Bailes J, Merrell R, O'Leary S: Stereotactic laser thermal ablation of recurrent posterior fossa metastatic lesion: description of new technology for infratentorial tumors refractory to conventional therapies. Neuro Oncol 16 (Suppl 5):v162, 2014 (Abstract)

5. Gonzalez-Martinez J, Vadera S, Mullin J, Enatsu R, Alexopoulos AV, Patwardhan R, et al: Robot-assisted stereotactic laser ablation in medically intractable epilepsy: operative technique. Neurosurgery 10 (Suppl 2):167-173, 2014

6. Hawasli AH, Ray WZ, Murphy RKJ, Dacey RG, Leuthardt EC: Magnetic resonance imaging-guided focused laser interstitial thermal therapy for subinsular metastatic adenocarcinoma: technical case report. Neurosurg 70 (2 Suppl Operative):332-338, 2012

7. Kangasniemi M, McNichols RJ, Bankson JA, Gowda A, Price RE, Hazle JD: Thermal therapy of canine cerebral tumors using a $980 \mathrm{~nm}$ diode laser with MR temperaturesensitive imaging feedback. Lasers Surg Med 35:41-50, 2004

8. Lefranc M, Capel C, Pruvot-Occean AS, Fichten A, Desenclos C, Toussaint P, et al: Frameless robotic stereotactic biopsies: a consecutive series of 100 cases. J Neurosurg 122:342-352, 2015

9. Lefranc M, Le Gars D: Robotic implantation of deep brain stimulation leads, assisted by intra-operative, flat-panel CT. Acta Neurochir (Wien) 154:2069-2074, 2012

10. Mattei TA, Rodriguez AH, Sambhara D, Mendel E: Current state-of-the-art and future perspectives of robotic technology in neurosurgery. Neurosurg Rev 37:357-366, 2014

11. Medvid R, Ruiz A, Komotar RJ, Jagid JR, Ivan ME, Quencer RM, et al: Current applications of MRI-guided laser interstitial thermal therapy in the treatment of brain neoplasms and epilepsy: a radiological and neurosurgical overview. AJNR Am J Neuroradiol 36:1998-2006, 2015

12. Mohammadi AM, Hawasli AH, Rodriguez A, Schroeder JL, Laxton AW, Elson P, et al: The role of laser interstitial thermal therapy in enhancing progression-free survival of difficult-to-access high-grade gliomas: a multicenter study. Cancer Med 3:971-979, 2014

13. Rahmathulla G, Recinos PF, Valerio JE, Chao S, Barnett GH: Laser interstitial thermal therapy for focal cerebral radiation necrosis: a case report and literature review. Stereotact Funct Neurosurg 90:192-200, 2012

14. Rao MS, Hargreaves EL, Khan AJ, Haffty BG, Danish SF: Magnetic resonance-guided laser ablation improves local control for postradiosurgery recurrence and/or radiation necrosis. Neurosurgery 74:658-667, 2014

15. Ringel F, Ingerl D, Ott S, Meyer B: VarioGuide: a new frameless image-guided stereotactic system-accuracy study and clinical assessment. Neurosurgery 64 (5 Suppl 2):365373, 2009

16. Sadraei NH, Dahiya S, Chao ST, Murphy ES, Osei-Boateng $\mathrm{K}$, Xie H, et al: Treatment of cerebral radiation necrosis with bevacizumab: the Cleveland clinic experience. Am J Clin Oncol 38:304-310, 2015

17. Sanai N, Polley MY, McDermott MW, Parsa AT, Berger MS: An extent of resection threshold for newly diagnosed glioblastomas. J Neurosurg 115:3-8, 2011

18. Siu A, Wind JJ, Iorgulescu JB, Chan TA, Yamada Y, Sherman JH: Radiation necrosis following treatment of high grade glioma-a review of the literature and current understanding. Acta Neurochir (Wien) 154:191-201, 2012

19. Torres-Reveron J, Tomasiewicz HC, Shetty A, Amankulor NM, Chiang VL: Stereotactic laser induced thermotherapy (LITT): a novel treatment for brain lesions regrowing after radiosurgery. J Neurooncol 113:495-503, 2013

\section{Disclosures}

Dr. Vadera reports being a consultant for and receiving an honorarium from Medtech.

\section{Author Contributions}

Conception and design: Vadera, Tran, Gill, Hsu. Acquisition of data: Vadera, Tran, Gill, Hsu. Analysis and interpretation of data: Vadera, Hsu. Drafting the article: all authors. Critically revising the article: all authors. Reviewed submitted version of manuscript: Vadera, Chan, Hsu. Administrative/technical/material support: Vadera. Study supervision: Vadera.

\section{Correspondence}

Sumeet Vadera, Department of Neurological Surgery, UC Irvine Medical Center, 101 The City Dr. South, Orange, CA 92868. email:svadera1@uci.edu. 